\title{
Studies on anti-hypercholesterolemic activity of Indanyl tetrazoles
}

\author{
Adak M \\ Department of Biochemistry, National Medical College and Teaching Hospital, Birgunj, Nepal. \\ Correspondence to: Dr. Manoranjan Adak, Department of Biochemistry, National Medical College and Teaching Hospital, \\ Birgunj, Nepal. \\ Email:manoranjanadak@rediffmail.com,itsmradak@gmail.com
}

\begin{abstract}
Introduction: Clinically useful non-steroidal antihyperlipidemic drugs are now in the market for the treatment of patients suffering from hypercholesterolemia. Indan acids, which belong to non-steroidal aryl alkanoic acids class acids have been reported to possess varying degrees of cholesterol lowering activity. Keeping the above points in view exploration for better anti-hypercholesterolemic activity of indanyl tetrazole derivatives was undertaken.

Methods: Five-week-old male albino Charles Foster rats $(120 \pm 10 \mathrm{~g})$ for normocholesterolemic and seven-week-old rats $(130 \pm 10 \mathrm{~g})$ for hypercholesterolemic studies were used. On the 0-day and 14th day, tail vein blood was drawn from the overnight fasted animals respectively. Biochemical measurements were carried out using biochemical kits (Span, India) and an automated biochemical analyzer (Vitros-250, Johnson and Johnson Co. USA).

Results: The compound - V and VI exhibited almost same significant level of cholesterol lowering $(\mathrm{p}<0.01)$ activity. On closer look its reveal that the compound-VI exhibit triglyceride lowering $(\mathrm{p}<0.01)$ activity among the groups around 14 days of treatment on normocholesterolemic rats. Test compound$\mathbf{V}(\mathrm{p}<0.05)$ and compound-VI $(\mathrm{p}<0.001)$ have significant anti-hyper-cholesterolemic activity while clofibrate has no such activity on hypercholesterolemic rats.

Conclusions: Indanyl tetrazole derivatives, 5-(62 -methoxyindan-12 -yl) methyltetrazole (V) and 5$(52,62$-dimethoxyindan-12 -yl) methyltetrazole (VI) were more promising than their analogs in respect to their anti-hypercholesterolemic activity.
\end{abstract}

\section{Introduction}

The most common clinically useful non-steroidal antihyperlipidemic drugs are now in the market each having its own limitation for chronic administration in the treatment of patients suffering from hypercholesterolemia. ${ }^{1-3}$ Among the non-steroidal agents, a series of aryl and aryloxy alkanoic acids were reported to possess maximum cholesterol lowering activity., ${ }^{4}$ Indan acids, which belong to nonsteroidal aryl alkanoic acids class, have assumed a special significance primarily because of its stereospecific structural framework making it a highly sensitive ring moiety towards biological action. ${ }^{6-8}$ The modified Indan ring system has been found to act as an inert carrier, which serves to hold biologically active functional moieties in a stereospecific manner ${ }^{9-13}$ Non-methoxy and methoxy substituted indan acids were reported to possess varying degrees of cholesterol lowering activity. ${ }^{14}$ It has also been reported that the smaller alkyl group $\left(-\mathrm{CH}_{3},-\mathrm{C}_{2} \mathrm{H}_{5}\right)$ at the á-carbon in the acetic acid moiety exhibited hypolipidemic activity. ${ }^{15-17}$ Keeping the above points in view, exploration for better anti-hypercholesterolemic activity of indanyl tetrazole derivatives was undertaken. It is already established that tetrazole, an aromatic azapyrrole group, is metabolically stable and encouraging anti-inflammatory activity has been noted. ${ }^{18-21-2}$ Further, indanyl tetrazoles were undertaken for cholesterol lowering study in normocholesterolemic and hypercholesterolemic animal models against standard drug 
clofibrate. These compounds are:<smiles>[X]c1ccc2c(c1)C(CCCCC)CC2</smiles><smiles>c1nnn[nH]1</smiles>

Where,

5-(Indan-12 -yl) tetrazole (I)

$\mathrm{X}=\mathrm{Y}=\mathrm{H}, \mathrm{n}=0$

5-(62 -methoxyindan-12 -yl)

tetrazole (II)

5-(52, 62 -dimethoxyindan

-12 -yl) tetrazole (III)

5-(Indan-12-yl)

methyltetrazole (IV)

5-(62 -methoxyindan-12 -yl)

methyltetrazole $(\mathbf{V})$

5-(52, 62 -dimethoxyindan

-12 -yl) methyltetrazole (VI

\section{Methods}

This study was conducted in the was conducted in Medicinal chemistry lab, dept. of pharmaceutical technology, Jadavpur university, Kolkata.

Indanyl tetrazoles, all biochemical kits (Span, India), standard drug clofibrate(IP), cholesterol(Sigma USA), sodium cholate ( Sigma, USA) were used for this study. ${ }^{22,23}$ Chemicals and solvent (EMark, India) used in this study were of analytical grade.

Normocholesterolemic: Five-week old male albino Charles Foster rats weighing $120 \pm 10 \mathrm{~g}$ were obtained from the animal house of the Department of Pharmaceutical Technology, Division of Medicinal Chemistry, Jadavpur University, Kolkata, India. Each group consisted of six animals .They were acclimatized to the laboratory environment (temperature $25^{\circ} \mathrm{C}+$ or - , $70-80 \%$ relative humidity and 12-hours lightdark cycle) for at least one week. They were fed standard laboratory diets (Gold Mohur, Hindustan Lever Ltd., Mumbai, India) and clean tap water ad libitum. ${ }^{24}$ All procedures involving animals were performed in accordance with the guidelines of the National Institutes of Health on the use and care of laboratory animals. ${ }^{25}$ Experimental procedures were also examined and approved by internal ethical committee for animal welfare.

Hypercholesterolemic: Seven week old male albino Charles Foster rats weighing $130 \pm 10$ were used. High cholesterol diet was prepared from laboratory standard diet by adding $1 \%$ cholesterol and $0.5 \%$ sodium cholate. Six animals in each groups were fed on high cholesterol diet for 10 days. ${ }^{26}$
Test compounds were dissolved in distilled water and the $\mathrm{pH}$ of the solution was adjusted to 7.5. The test drugs and clofibrate (aqueous solution) were then administered orally with the aid of a cannula at a dose of $50 \mathrm{mg} / \mathrm{kg}$ body weight for 14 days. Rats in the control group received $0.5 \mathrm{ml}$ the drug-free vehicle.

On the 0-day and 14th day, tail vein blood was drawn from the overnight fasted animals respectively. For biochemical study, blood (2-3 ml) was collected in non-heparinized $10 \mathrm{ml}$ test tube and was centrifuged at $1800 \mathrm{rpm}$ at $4^{\circ} \mathrm{C}$ for 10 minutes, and the serum was stored at $-20^{\circ} \mathrm{C}$ until analyzed.

The biochemical parameters were estimated at 0 day (before drug treatment) and 14th day after completion of drug treatment included serum total cholesterol, triglyceride, and high-density lipoprotein (HDL). Low-density lipoprotein (LDL) and very low-density lipoprotein (VLDL) fractions were calculated as:

i) $\quad$ VLDL $=$ triglycerides $/ 5$

ii) $\quad \mathrm{LDL}=$ total cholesterol $-(\mathrm{HDL}$ cholesterol + VLDL cholesterol) respectively. ${ }^{27}$ Biochemical measurements were carried out using biochemical kits (Span, India) and an automated biochemical analyzer (Vitros-250, Johnson and Johnson Co. USA).

The results are presented as mean $\pm \mathrm{SE}$. The results were analyzed statistically by using one-way analysis of variance (ANOVA) followed by Dunnett's $t$-test. P values less than 0.05 were considered as significant. All analysis was performed using SPSS 10.0 statistical software.

\section{Results}

The effects of oral administration of indanyl tetrazoles on serum total cholesterol, triglyceride and lipoproteins of normocholesterolemic rats are analyzed. It is seen that serum cholesterol and triglyceride level in normal rats did not reveal any significant decrease during the experimental period of 14 days when compared with standard drug, clofibrate (Table 1). The compound - V and VI exhibited almost same significant level of cholesterol lowering $(\mathrm{p}<0.01)$ activity. The compound-VI exhibits significant triglyceride lowering $(\mathrm{p}<0.01)$ activity among the groups at around 14 days of treatment. The clofibrate treated animals caused a progressive decrease in serum cholesterol as well as triglyceride $(\mathrm{p}<0.001)$ that were found to be maximum after 14 days of treatment. Test compounds as well as standard drug, clofibrate have no effect on HDL cholesterol whereas VLDL cholesterol lowering activity was found only in clofibrate treated animals . Slight LDL- cholesterol lowering effect were found in test compound-VI $(\mathrm{p}<0.01)$ and in 
clofibrate $(<0.05)$.

Table 1: Effects of indanyl tetrazoles on serum total cholesterol, triglyceride and lipoproteins in normo-cholesterolemic rats.

\begin{tabular}{|c|c|c|c|c|c|c|c|c|c|c|}
\hline \multirow[t]{2}{*}{ Group } & \multicolumn{2}{|c|}{$\begin{array}{c}\text { Serum total } \\
\text { cholesterol(mg/dl) }\end{array}$} & \multicolumn{2}{|c|}{$\begin{array}{l}\text { Serum } \\
\text { triglyceride } \\
(\mathbf{m g} / \mathbf{d l})\end{array}$} & \multicolumn{2}{|c|}{$\begin{array}{c}\text { HDL-cholesterol } \\
(\mathrm{mg} / \mathrm{dl})\end{array}$} & \multicolumn{2}{|c|}{$\begin{array}{c}\text { LDL - cholesterol } \\
(\mathrm{mg} / \mathrm{dl})\end{array}$} & \multicolumn{2}{|c|}{$\begin{array}{c}\text { VLDL-cholesterol } \\
\text { (mg/dl) }\end{array}$} \\
\hline & O day & $14^{\text {th }}$ day & 0 day & $14^{\text {th }}$ day & 0 day & $14^{\text {th }}$ day & 0 day & $14^{\text {th }}$ day & O day & $14^{\text {th }}$ day \\
\hline Comp-I & $\begin{array}{l}83.33 \\
\pm 0.62\end{array}$ & $\begin{array}{l}81.00 \\
\pm 0.49\end{array}$ & $\begin{array}{l}30.33 \\
\pm 0.42\end{array}$ & $\begin{array}{l}29.00 \\
\pm 0.36\end{array}$ & $\begin{array}{l}24.60 \\
\pm 1.26\end{array}$ & $\begin{array}{l}25.17 \\
\pm 1.11\end{array}$ & $\begin{array}{l}52.60 \\
\pm 1.57\end{array}$ & $\begin{array}{l}50.03 \\
\pm 1.49\end{array}$ & $\begin{array}{l}6.07 \\
\pm 0.08\end{array}$ & $\begin{array}{l}5.80 \\
\pm 0.07\end{array}$ \\
\hline Comp-II & $\begin{array}{r}81.67 \\
\pm 0.67\end{array}$ & $\begin{array}{l}77.83 \\
\pm 0.75\end{array}$ & $\begin{array}{l}31.83 \\
\pm 0.48\end{array}$ & \begin{tabular}{|l|}
30.33 \\
\pm 0.49
\end{tabular} & $\begin{array}{l}24.00 \\
\pm 1.24\end{array}$ & $\begin{array}{l}25.00 \\
\pm 0.93\end{array}$ & $\begin{array}{l}50.93 \\
\pm 1.40\end{array}$ & $\begin{array}{l}46.93 \\
\pm 1.35\end{array}$ & $\begin{array}{r}6.37 \\
\pm 0.09\end{array}$ & $\begin{array}{c}6.07 \\
\pm 0.09\end{array}$ \\
\hline Comp-III & $\begin{array}{l}79.17 \\
\pm 0.87\end{array}$ & $\begin{array}{c}75.00 \\
\pm 1.09^{\mathrm{a}}\end{array}$ & $\begin{array}{c}28.33 \\
\pm 0.49\end{array}$ & $\begin{array}{l}26.50 \\
\pm 0.34\end{array}$ & $\begin{array}{l}26.33 \\
\pm 1.33\end{array}$ & $\begin{array}{l}27.17 \\
\pm 1.01\end{array}$ & $\begin{array}{l}47.00 \\
\pm 1.99\end{array}$ & $\begin{array}{l}42.86 \\
\pm 1.76\end{array}$ & $\begin{array}{l}5.67 \\
\pm 0.09\end{array}$ & $\begin{array}{l}5.30 \\
\pm 0.07\end{array}$ \\
\hline Comp-IV & $\begin{array}{l}82.17 \\
\pm 0.48\end{array}$ & $\begin{array}{l}78.33 \\
\pm 0.80^{\mathrm{b}}\end{array}$ & $\begin{array}{l}30.17 \\
\pm 0.79\end{array}$ & $\begin{array}{l}29.17 \\
\pm 0.70\end{array}$ & $\begin{array}{l}24.67 \\
\pm 0.88\end{array}$ & $\begin{array}{l}26.33 \\
\pm 1.12\end{array}$ & $\begin{array}{l}51.48 \\
\pm 1.17\end{array}$ & $\begin{array}{l}46.83 \\
\pm 0.92\end{array}$ & $\begin{array}{r}6.03 \\
\pm 0.16\end{array}$ & $\begin{array}{l}5.83 \\
\pm 0.14\end{array}$ \\
\hline Comp-V & $\begin{array}{l}78.33 \\
\pm 0.99\end{array}$ & $\begin{array}{l}72.67 \\
\pm 0.54^{\mathrm{c}}\end{array}$ & $\begin{array}{l}30.83 \\
\pm 0.79\end{array}$ & $\begin{array}{l}29.00 \\
\pm 0.89^{b}\end{array}$ & $\begin{array}{l}25.40 \\
\pm 1.72\end{array}$ & $\begin{array}{l}27.00 \\
\pm 1.48\end{array}$ & $\begin{array}{l}46.32 \\
\pm 1.22\end{array}$ & $\begin{array}{l}41.00 \\
\pm 1.78\end{array}$ & $\begin{array}{l}6.28 \\
\pm 0.14\end{array}$ & $\begin{array}{r}6.00 \\
\pm 0.23\end{array}$ \\
\hline Comp-VI & $\begin{array}{l}79.17 \\
\pm 0.48\end{array}$ & $\begin{array}{l}71.17 \\
\pm 0.89^{c}\end{array}$ & $\begin{array}{l}31.16 \\
\pm 0.79\end{array}$ & $\begin{array}{l}28.67 \\
\pm 0.80^{\mathrm{b}}\end{array}$ & $\begin{array}{l}26.57 \\
\pm 1.07\end{array}$ & $\begin{array}{l}28.57 \\
\pm 1.21\end{array}$ & $\begin{array}{l}47.09 \\
\pm 1.18\end{array}$ & $\begin{array}{l}38.43 \\
\pm 1.35^{\mathrm{a}}\end{array}$ & $\begin{array}{l}6.10 \\
\pm 0.16\end{array}$ & \begin{tabular}{|l|}
5.63 \\
\pm 0.13
\end{tabular} \\
\hline Clofibrate & $\begin{array}{l}78.00 \\
\pm 0.58\end{array}$ & $\begin{array}{l}64.50 \\
\pm 0.89^{d}\end{array}$ & $\begin{array}{l}35.50 \\
\pm 1.26\end{array}$ & $\begin{array}{l}29.00 \\
\pm 1.03^{\mathrm{d}}\end{array}$ & $\begin{array}{l}25.67 \\
\pm 1.80\end{array}$ & $\begin{array}{l}26.17 \\
\pm 1.64\end{array}$ & $\begin{array}{l}43.57 \\
\pm 2.87\end{array}$ & $\begin{array}{l}32.53 \\
\pm 2.18^{\mathrm{b}}\end{array}$ & $\begin{array}{r}7.10 \\
\pm 0.25\end{array}$ & $\begin{array}{r}5.80 \\
\pm 0.21^{\mathrm{a}}\end{array}$ \\
\hline Control & $\begin{array}{l}78.50 \\
\pm 0.76\end{array}$ & $\begin{array}{l}81.17 \\
\pm 0.91\end{array}$ & $\begin{array}{l}33.50 \\
\pm 1.39\end{array}$ & \begin{tabular}{|l|}
34.83 \\
\pm 1.49
\end{tabular} & $\begin{array}{l}24.17 \\
+1.35\end{array}$ & $\begin{array}{l}25.17 \\
\pm 1.05\end{array}$ & $\begin{array}{l}47.63 \\
\pm 2.04\end{array}$ & $\begin{array}{r}49.33 \\
\pm 1.71\end{array}$ & $\begin{array}{r}6.70 \\
\pm 0.25\end{array}$ & \begin{tabular}{|r}
6.97 \\
\pm 0.30
\end{tabular} \\
\hline
\end{tabular}

a-d Probability values ( calculated as compared to 0 day within group using Dunnett $t$-test) $\mathrm{a}<0.25, \mathrm{~b}<0.05 \mathrm{c}<0.01, \mathrm{~d}<0.001$. Each value represents mean \pm SEM, $(n=6)$

The compound-V $(\mathrm{p}<0.05)$ and compound-VI $(\mathrm{p}<0.001)$ have significant anti-hyper-cholesterolemic activity while clofibrate has no such activity (Table 2). Interesting observation is that compound-VI is also exhibit significant triglyceride lowering activity $(\mathrm{p}<0.01)$ among the test agents but none of them showed better triglyceride lowering activity in comparison to that of clofibrate $(\mathrm{p}<0.001)$. The levels of cholesterol in serum HDL, LDL and VLDL fractions of hypercholesterolemic rats were estimated (Table 2). The results showed that test compounds exhibit variable lipoprotein lowering activity, and a few among them have significant activity. HDL cholesterol level was increased and LDL level was decreased by the test compound- $\mathbf{V}$ $(\mathrm{p}<0.01)$ and-VI $(\mathrm{p}<0.001)$ which were statistically significant. However, VLDL cholesterol level was significantly reduced only by clofibrate $(\mathrm{p}<0.05)$.

No enlargements of liver were found in this biomodel when treated with test agents but apparent body weights gain were found which were statistically insignificant. 
Anti-hypercholesterolemic activity

Table 2: Effects of indanyl tetrazoles on serum total cholesterol, triglyceride and lipoproteins in hypercholesterolemic rats.

\begin{tabular}{|c|c|c|c|c|c|c|c|c|c|c|}
\hline \multirow[t]{2}{*}{ Group } & \multicolumn{2}{|c|}{$\begin{array}{l}\text { Serum total } \\
\text { cholesterol }(\mathrm{mg} / \mathrm{dl})\end{array}$} & \multicolumn{2}{|c|}{ triglyceride $(\mathrm{mg} / \mathrm{dl})$} & \multicolumn{2}{|c|}{$\begin{array}{l}\text { HDL-cholesterol } \\
(\mathrm{mg} / \mathrm{dl})\end{array}$} & \multicolumn{2}{|c|}{$\begin{array}{l}\text { LDL- cholesterol } \\
(\mathrm{mg} / \mathrm{dl})\end{array}$} & \multicolumn{2}{|c|}{$\begin{array}{c}\text { VLDL-cholesterol } \\
(\mathrm{mg} / \mathrm{dl})\end{array}$} \\
\hline & 0 day & $14^{\text {th }}$ day & 0 day & $14^{\text {th }}$ day & 0 day & $14^{\text {th }} \mathrm{day}$ & O day & $14^{\text {th }}$ day & & $14^{\text {th }}$ day \\
\hline Comp-I & $\begin{array}{l}99.17 \\
\pm 0.48\end{array}$ & $\begin{array}{l}94.67 \\
\pm 0.56\end{array}$ & $\begin{array}{l}48.33 \\
\pm 0.88\end{array}$ & $\begin{array}{l}45.17 \\
\pm 1.11\end{array}$ & $\begin{array}{l}26.50 \\
\pm 1.23\end{array}$ & $\begin{array}{l}28.33 \\
\pm 1.08\end{array}$ & $\begin{array}{l}63.13 \\
\pm 1.08\end{array}$ & $\begin{array}{l}60.00 \\
\pm 1.61\end{array}$ & $\begin{array}{l}9.67 \\
\pm 0.17\end{array}$ & $\begin{array}{l}9.20 \\
\pm 0.22\end{array}$ \\
\hline Comp-II & $\begin{array}{l}98.00 \\
\pm 0.52\end{array}$ & $\begin{array}{l}92.67 \\
\pm 0.72\end{array}$ & $\begin{array}{l}49.67 \\
\pm 0.67\end{array}$ & $\begin{array}{l}46.50 \\
\pm 0.67\end{array}$ & $\begin{array}{l}26.83 \\
\pm 0.79\end{array}$ & $\begin{array}{l}28.67 \\
\pm 0.62\end{array}$ & $\begin{array}{l}61.23 \\
\pm 0.85\end{array}$ & $\begin{array}{l}58.20 \\
\pm 1.66\end{array}$ & $\begin{array}{l}9.93 \\
\pm 0.13\end{array}$ & $\begin{array}{l}9.27 \\
\pm 0.15\end{array}$ \\
\hline Comp-III & $\begin{array}{l}99.00 \\
\pm 0.79\end{array}$ & $\begin{array}{l}92.50 \\
\pm 0.43^{\text {a }}\end{array}$ & $\begin{array}{l}49.00 \\
\pm 0.97\end{array}$ & $\begin{array}{l}45.67 \\
\pm 1.05^{\mathrm{a}}\end{array}$ & $\begin{array}{l}28.17 \\
\pm 0.79\end{array}$ & $\begin{array}{l}32.00 \\
\pm 0.78^{\mathrm{a}}\end{array}$ & $\begin{array}{l}62.20 \\
\pm 0.56\end{array}$ & $\begin{array}{l}50.70 \\
\pm 0.81^{\mathrm{a}}\end{array}$ & $\begin{array}{l}9.80 \\
\pm 0.19\end{array}$ & $\begin{array}{l}9.13 \\
\pm 0.21\end{array}$ \\
\hline Comp-IV & $\begin{array}{l}97.83 \\
\pm 0.79\end{array}$ & $\begin{array}{l}92.17 \\
\pm 0.95^{\mathrm{a}}\end{array}$ & $\begin{array}{l}49.83 \\
\pm 0.60\end{array}$ & $\begin{array}{l}46.50 \\
\pm 0.56^{\mathrm{a}}\end{array}$ & $\begin{array}{l}26.00 \\
\pm 1.24\end{array}$ & $\begin{array}{l}30.17 \\
\pm 1.25^{\mathrm{b}}\end{array}$ & $\begin{array}{l}61.93 \\
\pm 1.31\end{array}$ & $\begin{array}{l}58.73 \\
\pm 1.86^{\mathrm{a}}\end{array}$ & $\begin{array}{l}9.97 \\
\pm 0.12\end{array}$ & $\begin{array}{l}9.30 \\
\pm 0.11\end{array}$ \\
\hline Comp-V & $\begin{array}{l}97.83 \\
\pm 0.99\end{array}$ & $\begin{array}{l}89.67 \\
\pm 0.42^{b}\end{array}$ & $\begin{array}{l}48.67 \\
\pm 0.72\end{array}$ & $\begin{array}{l}45.83 \\
\pm 0.87^{\mathrm{a}}\end{array}$ & $\begin{array}{l}27.83 \\
\pm 1.42\end{array}$ & $\begin{array}{l}33.50 \\
\pm 1.29^{\mathrm{c}}\end{array}$ & $\begin{array}{l}58.60 \\
\pm 0.71\end{array}$ & $\begin{array}{l}46.83 \\
\pm 1.99^{\mathrm{b}}\end{array}$ & $\begin{array}{l}9.80 \\
\pm 0.19\end{array}$ & $\begin{array}{l}9.67 \\
\pm 0.32\end{array}$ \\
\hline Comp-VI & $\begin{array}{l}97.50 \\
\pm 0.76\end{array}$ & $\begin{array}{l}86.67 \\
\pm 1.31^{\mathrm{d}}\end{array}$ & $50.00 \pm 0.86$ & $\begin{array}{l}45.67 \\
\pm 0.76^{\mathrm{c}}\end{array}$ & $\begin{array}{l}26.17 \\
\pm 1.54\end{array}$ & $\begin{array}{l}34.50 \\
\pm 1.46^{\mathrm{d}}\end{array}$ & $\begin{array}{l}57.90 \\
\pm 1.07\end{array}$ & $\begin{array}{l}43.07 \\
\pm 0.66^{\mathrm{c}}\end{array}$ & $\begin{array}{l}10.00 \\
\pm 0.17\end{array}$ & $\begin{array}{l}9.13 \\
\pm 0.15\end{array}$ \\
\hline Clofibrate & $\begin{array}{l}98.50 \\
\pm 0.82\end{array}$ & $\begin{array}{l}97.17 \\
\pm 0.75\end{array}$ & $\begin{array}{l}50.00 \\
\pm 0.58\end{array}$ & $\begin{array}{l}41.00 \\
\pm 0.37^{\mathrm{d}}\end{array}$ & $\begin{array}{l}26.67 \\
\pm 1.56\end{array}$ & $\begin{array}{l}27.33 \\
\pm 1.61\end{array}$ & $\begin{array}{l}60.40 \\
\pm 2.23\end{array}$ & $\begin{array}{l}59.60 \\
\pm 2.49\end{array}$ & $\begin{array}{l}9.83 \\
\pm 0.23\end{array}$ & $\begin{array}{l}8.20 \\
\pm 0.07^{\mathrm{b}}\end{array}$ \\
\hline Normal & $\begin{array}{l}79.17 \\
\pm 0.58\end{array}$ & $\begin{array}{l}83.50 \\
\pm 0.85\end{array}$ & $\begin{array}{l}37.17 \\
\pm 0.95\end{array}$ & $\begin{array}{l}38.67 \\
\pm 0.76\end{array}$ & $\begin{array}{l}24.17 \\
\pm 1.35\end{array}$ & $\begin{array}{l}25.27 \\
\pm 1.08\end{array}$ & $\begin{array}{l}48.63 \\
\pm 2.04\end{array}$ & $\begin{array}{l}49.35 \\
\pm 1.72\end{array}$ & $\begin{array}{l}6.73 \\
\pm 0.25\end{array}$ & $\begin{array}{l}6.97 \\
\pm 0.30\end{array}$ \\
\hline
\end{tabular}

${ }^{\mathrm{a}-\mathrm{d}}$ Probability values ( calculated as compared to 0 day within group using Dunnett $t$-test) $\mathrm{a}<0.025, \mathrm{~b}<0.05, \mathrm{c}<0.01, \mathrm{~d}<$ 0.001. Each value represents mean \pm SEM, $(n=6)$

\section{Discussion}

The results of pharmacological screening on normocholesterolemic and hypercholesterolemic rats indicated that indanyl tetrazoles exhibited varying degrees of anti-hypercholesterolemic activity. However, the methoxy substitution in the indan nucleus has a better edge over non-methoxy derivatives when compared with standard drug clofibrate. Dimethoxy substituted indanyl tetrazoles showed an appreciable antihypercholesterolemic activity and which were found to be statistically significant. Earlier works had also established biological activity reside in methoxy substituted indan acids, which corroborate our present studies. ${ }^{16}$ Thus noticeable antihypercholesterolemic activity of $5^{\prime}-\mathrm{OCH}_{3}$ and $6^{\prime}-\mathrm{OCH}_{3}$ substituted indan with tetrazoles group at acid moiety were possible either due to their favorable lipophilic and ionic character or better drug receptor interaction or to their biotransformation or conversion to active metabolite in the biological system. So, they necessitate further detailed studies regarding their pharmacological profile as well as metabolism.

High levels of HDL cholesterol and low levels of LDL cholesterol have positive correlation with cardiovascular system. HDL inhibits the uptake of LDL by arterial wall and facilitates the transport of cholesterol from peripheral tissue to the liver where it is catabolised and excreted out of the body. ${ }^{28}$ Our findings regarding lipoprotein suggest that indanyl tetrazole particularly dimethoxy indanyl tetrazoles possesses positive effect on the circulation of body cholesterol.

Changes in body weight and organ weights are used as an indicator of adverse effect of drugs and chemicals. ${ }^{29,30}$ In the present study, treatment resulted that an average body weights of all experimental animals were within the normal 
range throughout the experimental period. These observations suggesting that the test agents are less toxic hence have no adverse effect.

\section{Conclusions}

Indanyl tetrazole derivatives exhibited variable antihypercholesterolemic activity. However, the activity of 5(62 -methoxyindan-12 -yl) methyltetrazole (V) and 5-(52,62 -dimethoxyindan-12 -yl) methyltetrazole (VI) were more promising than their analogs. From these observations, it seems likely that further modification of structural frame of these indanyl tetrazoles may improve the antihypercholesterolemic activity. Studies are also needed towards metabolism, and toxic manifestation of the potent compounds. Work in this direction is in progress and will be reported elsewhere.

\section{Acknowledgements}

Author, Dr. M R Adak is thankful to Council of Scientific and Industrial Research, New Delhi for their financial assistance and acknowledges the guidance throughout this by the late Prof. S C Lahiri, my friend to whom I dedicate this paper.

\section{References}

1. Kenneth HK, Erwin KK. Drug Facts and Comparisons: St. Louis, Facts and Comparisons, 57th ed. Wolters Kluwer Company, 2003. p. 2496.

2. Kline and Company (www.klinegroup.com). U.S. Cholesterol-Lowering Drugs 2005: Rx Market Analysis and Switch Forecasts.

3. Nicholas AB, Nicki RC, Brain RW, John AAH. Cardiovascular disease: In Davidson's principles and practice of medicine.20th ed. Churchill Livingstone, New York, 2006. p. 520-644.

4. Garattini S, Paoletti R, Bitti L, Grossi E, Vertua R. In: Drug Affecting lipid Metabolism. Elsevier, Amsterdam, 1996.p. 144.

5. Schacht E. Hypolipidemic aryloxyacetic acids: Topics in Current chemistry. Vol 72, Springer Berlin, Heidelberg, 2006. p. 99-123.

6. Ganellin CR. Indan and indene derivatives of biological interest. In advance in Drug Research, Harper WJ, Simmond AB editor, Vol-4, London: Academic Press, 1967.p.163.

7. Pingali H, Jain M, Shah S, Basu S, Makadia P, Goswami $\mathrm{A}$,et al. Discovery of a highly orally bioavailable $c$-5-
[6-(4-Methanesulfonyloxyphenyl)hexyl]-2-methyl-1,3dioxane- $r$-carboxylic acid as a potent hypoglycemic and hypolipidemic agent. Bioorg Med. Che.Letter.2008; 18(20): 5586-5590.

8. Allen GR Jr, Littell R, McEvoy FJ, Sloboda AE. 5substituted -1- indan carboxylic acids as potential antiinflammatory agents. J Med.Chem. 1972; 15(9): 934-937.

9. Sharrma M, Ray SM. Synthesis and Biological Evaluation of Amide Derivatives of (6- Chloro-2, 3dihydro- $1 H$-inden-1-yl) acetic Acid as Potential Antiinflammatory Agents with Lower Gastrointestinal Toxicity. Chem Pharma Bull.2008; 56(5): 626-634.

10. Lahiri SC, Gupta JK. Studies on indan acids as potential oral hypoglycemic agent. J Ind Chem Soc. 1976; 53: 10411043.1.References and further reading may be available for this article. To view references and further reading you must purchase this article.

11. Roy A, Gupta JK, Lahiri SC. Further studies on antiinflammatory activity of two potent indan-1-acetic acids. Ind J Physiol Pharmac. 1982; 26: 207-214.

12. Ray SM, Lahiri SC. Investigation on synthesis, hypotensive activity and highly selective adrenergic antagonistic activity of some simple and substituted indan derivatives. J Ind Chem Soc.1991; 68: 546-555.

13. Mukhopadhyay A, Roy A, Lahiri SC. Prostaglandin biosynthesis inhibitory activity of some indan-1-acids in relation to their anti-inflammatory activity and ulcerogenic potency. Ind J Expt Biol.1994; 31: 392-394.

14. Bacher SC, Lahiri SC. Plant growth regulatory activity some simple and methoxy substituted indan-1-carboxy acids on mustard (Brassica juncea $L$ ) seedlings. Ind J Expt Biol.1997; 32: 598-599.

15. Adak M, Lahiri SC. Studies on hypocholesterolemic activity of some simple and substituted indan-1- acetic acids. Part-I. Indian Drugs. 1995; 32: 583-585.

16. Adak M. Gupta JK. Synthesis and antihypercholesterolemic activity of á-alkyl substituted indan-1-acetic acids. Asian J Chem.2006; 18(2): 13941400.

17. Adak M, Gupta JK. Hypolipidemic activity of indan-1acetic acids . Nepal Med Coll J.2006; 8(1): 22-26.

18. Fidgor SK, Von Wittenau MS. Metabolism of 5-(3pyridyl) tetrazole. J Med Chem.1967; 10: 1158-1159.

19. Ray SM, Lahiri SC. Studies on 5-(indan-12 -yl) tetrazoles as potential non-steroidal anti-inflammatory 
agents. J Ind Chem Soc.1990; 67: 324-326.

20. Bachar SC, Lahiri SC. Synthesis of chloro and bromo substituted 5-(indan-12 -yl) tetrazole and 5- (indan-12 -yl) methyltetrazole as possible analgesic agents. Pharmazie.2004; 59: 435-438.

21. Bepary S, Das, BK, Bachar SC, Kundu JK., Shamsurrof AS, Datta BK. Anti-inflammatory activity of indanyl tetrazole. Pak J Pharma Sci.2008; 21(3): 295-298.

22. Roy A, Gupta JK, Lahiri SC. Synthesis and antiinflammatory activity of a few 5-(indan-12 - yl) methyltetrazole. J Ind. Chem Soc.1983; 60: 377-380.

24. 23.Roy A, Lahiri SC. Studies on two novel antiinflammatory indanyl methyl tetrazoles. Ind J Physiol Pharmac.1985; 17: 63-66.Khanna N, Ray A, Alkondon M, Sen P. Effect of á-adrenoceptor antagonists and some related drugs on maximal electroshock seizures in mice. Ind J Exp Bio.1989; 27: 128-130.

25. National Institutes of Health Public Health Service Policy on the humane care and use of laboratory animals. Bethesda, MD: NIH, 2002

26. Kameo K, Asami Y, Ogawa K, Matusnaga T, Saito S, Tomisaw K, Sota K. Studies hypolipidemic agents. IV.3-[4-(phenylthio)-benzoxy] Propionic acid derivatives. Chem Pharma Bull.1989; 37: 1260-1267.

27. Keaney JFJ, Gaziano JM, Xu A, Frei B, CurranCelentano J, Shwaery GT, et al. Low-Dose a-Tocopherol Improves and High- Dose a-Tocopherol Worsens Endothelial Vasodilator Function in Cholesterol-Fed Rabbits. The J Clin Invest Inc. 1994; 93: 844-851.

28. Miller GJ, Miller NE. Plasma-high-density-lipoprotein concentration and development of ischemic heart disease. Lancet1975; 1: 16-19.

29. Tofovic SP, Jackson EK. Effects of long-term caffeine consumption on renal function in spontaneously hypertensive heart failure prone rats. J Cardio Pharmacol.1999; 33: 360-366.

30. Adak M, Gupta JK. Acute and Subacute Toxicity study with Hypolipidemic agent of á- Alkyl Substituted Indan1- Acetic Acids in mice. J Med Res. Med Sci.2009; 4(2): 345-350. 